\title{
Reformulating Task-Related Component Analysis for Reducing its Computational Complexity
}

This paper was downloaded from TechRxiv (https://www.techrxiv.org).

\section{LICENSE}

CC BY 4.0

SUBMISSION DATE / POSTED DATE

$11-01-2022$ / 14-01-2022

\section{CITATION}

Chiang, Kuan-Jung; Wong, Chi Man; Wan, Feng; Jung, Tzyy-Ping; Nakanishi, Masaki (2022): Reformulating Task-Related Component Analysis for Reducing its Computational Complexity. TechRxiv. Preprint. https://doi.org/10.36227/techrxiv.18131468.v1

$\mathrm{DOI}$ 


\title{
Reformulating Task-Related Component Analysis for Reducing its Computational Complexity
}

\author{
Kuan-Jung Chiang, Student Member, IEEE, Chi Man Wong, Feng Wan, Senior Member, IEEE, \\ Tzyy-Ping Jung, Fellow, IEEE, and Masaki Nakanishi, Senior Member, IEEE
}

\begin{abstract}
The task-related component analysis (TRCA) is a data-driven method for extracting reproducible components across multiple data segments from multivariate data. TRCA has been proven effective in enhancing the signal-to-noise ratio of neuroimaging data in previous studies. However, it requires a computational cost of $\mathcal{O}\left(N^{2}\right)$ to compute cross-covariance matrices, indicating that the computational time increases rapidly as the number of data segments $N$ increases. This study proposed to reduce the computational complexity to $\mathcal{O}(N)$ theoretically by reformulating the covariance computations required in TRCA. Simulation study that compared the original and proposed TRCA against synthetic data with various sizes (e.g., data segments, dimensions, and lengths) validated the computation reduction. The proposed reformulation would be important in practice especially when applying the TRCA to large-scale computations.
\end{abstract}

Index Terms-Biomedical data analysis, generalized eigenvalue problem, task-related component analysis

\section{INTRODUCTION}

$\mathbf{T}$ ASK-RELATED component analysis (TRCA) is a multivariate data-driven method that extracts underlying reproducible components from multiple data segments of multivariate data [1]. TRCA has been successfully used in neuroimaging studies to extract target brain activities. A neuroimaging experiment typically requires participants to complete given tasks multiple times while multiple sensors record timeseries data. TRCA uses inter-trial covariance maximization to find linear coefficients that maximize the consistency of transformed data across such task trials. A component extracted by this procedure is called a task-related component. TRCA can produce a higher signal-to-noise ratio (SNR) than the single-channel trial-averaging approach commonly used in biological data analysis due to the advantage of multi-channel measurement and analysis.

TRCA was first proposed by Tanaka et al. and demonstrated on synthetic data and functional near-infrared spectroscopy (fNIRS) data [1]. The authors validated the effectiveness of

This work is supported in part by Army Research Laboratory (W911NF2120154), and the US National Science Foundation (NCS-1734883, CBET-1935860, IP-1719130, and SMA-15409430, the Science and Technology Development Fund, Macau SAR (File no. 0045/2019/AFJ), the University of Macau Research Committee (MYRG projects 2016-00240-FST and 2017 00207-FST). (K. -J. Chiang and C. M. Wong contributed equally to the work. Corresponding auther: Masaki Nakanishi.)

K. -J. Chiang, M. Nakanishi, and T. -P. Jung are with the Swartz Center for Computational Neuroscience, Institute for Neural Computation, University of California San Diego, La Jolla, CA 92093, USA e-mail: masaki@sccn.ucsd.edu.

C. M. Wong and F. Wan are with the Department of Electrical and Computer Engineering, University of Macau, Taipa, Macau.
TRCA in extracting hemodynamic responses corresponding to finger-tapping tasks and removing task-unrelated artifacts in the fNIRS data [1], [2]. In addition to fNIRS data, TRCA was naturally used as a denoising method in electroencephalographic (EEG) studies because event-related EEG potentials (ERPs), especially evoked potentials, were time-locked and/or phase-locked to event onsets and thus highly consistent across task periods. To date, TRCA's most successful application is steady-state visual evoked potentials (SSVEPs). Nakanishi et al. used TRCA as a spatial filter for enhancing the SNR of SSVEPs in their brain-computer interface (BCI) study [3]. Since then, TRCA has been recognized as the most effective spatial-filtering method to implement a high-performance SSVEP-based BCI [4].

Following its success in biological data analysis, several extensions of TRCA have been proposed [5]-[7]. For example, Tanaka et al. proposed cross-correlation TRCA (xTRCA), which is a unified framework for analyzing event-related evoked and induced potentials [5]. In contrast to evoked potentials, induced potentials such as P300 are not phaselocked to event onsets [8], so they could be cancelled out by trial averaging. The xTRCA employs a temporal optimization to compensate for trial-by-trial latency variability, providing a better solution to enhance induced potentials than the conventional TRCA. Another extension is group TRCA (gTRCA) designed for group-level analysis [6]. The gTRCA finds linear coefficients that maximize not only trial-by-trial reproducibility within single subjects but also similarity across a group of subjects. In addition, an online version of TRCA, which recursively updates the solution for generalized eigenvalue problem, was also proposed toward its online applications such as BCIs [7].

Increasing variations and applications of the TRCA method has made its high computational cost impossible to ignore, especially in cases where a large dataset or repeated calculations are needed. First of all, in the conventional TRCA, a non-parametric statistical test based on resampling, in which eigenvalue decomposition needs to be performed repeatedly, is required to find reproducible task-related components [1], [2], [7], [9]. In addition, xTRCA also needs to compute TRCA multiple times to find the best temporal alignment across trials using a sliding window [5]. On the other hand, gTRCA solves eigenvalue decomposition on a large covariance matrix involving data from multiple subjects. Intuitively, as the number of task trials and data dimension increase, so does the computational cost of TRCA. Because TRCA's high computational complexity could hinder its real-world applications, 
a computationally inexpensive algorithm to solve TRCA is desired.

This study aims to reformulate the algorithm of TRCA to reduce its computational complexity. The key idea is to reverse the order of multiplication and summation in computing covariance matrices. In the original TRCA, the algorithm computes the sum of cross-covariance matrices (i.e., matrix multiplication) of multi-dimensional data between each pair of trials. As a result, the number of matrix multiplications is proportional to the square of the number of trials. We found that the final covariance matrix obtained by this procedure is mathematically equal to the auto-covariance matrix of the sum of the data across trials. Because the auto-covariance matrix of the sum of trials requires only a single matrix multiplication, the computational complexity would be significantly reduced. To validate the effectiveness of the proposed reformulation on reducing the computational time, we performed a computer simulation using synthetic data with varying data dimensions.

In the following sections, we will first give a brief introduction to the original TRCA and then describe our proposed reformulation (it is called fast TRCA here). Finally, we will present a series of computer simulations, in which the original and the proposed TRCAs were applied to synthetic data, and discussed the study results. The code may be downloaded from https://github.com/mnakanishi/TRCA-SSVEP.

\section{TASK-RELATED COMPONENT ANALYSIS}

TRCA aims to extract task-related components efficiently by maximizing their reproducibility during task periods [1], [3]. Considering an observed multi-dimensional signals $\boldsymbol{x}=$ $(x)_{i j} \in \mathbb{R}^{I \times J}$, TRCA finds a linear coefficient vector $\boldsymbol{w} \in$ $\mathbb{R}^{I}$ to maximize inter-trial correlation of its projections $\boldsymbol{y}=$ $\boldsymbol{w}^{T} \boldsymbol{x}$, which is called a task-related component (see Algorithm 1). Here, $i$ represents the index of data dimension (e.g., the number of sensors in neuroimaging data), $I$ is the number of dimensions, $j$ is the index of sample points, and $J$ is the number of sample points in a data epoch. The $n$-th trial in the observed EEG signals and task-related components are described as $\boldsymbol{x}^{(n)} \in \mathbb{R}^{I \times J}$ and $\boldsymbol{y}^{(n)} \in \mathbb{R}^{J}$, respectively.

The covariance $C_{n_{1}, n_{2}}$ between the $n_{1}$-th and the $n_{2}$-th trials of $\boldsymbol{y}$ is described as:

$$
\begin{aligned}
C_{n_{1}, n_{2}} & =\operatorname{Cov}\left(\boldsymbol{y}^{\left(n_{1}\right)}, \boldsymbol{y}^{\left(n_{2}\right)}\right) \\
& =\sum_{i_{1}, i_{2}=1}^{I} w_{i_{1}} w_{i_{2}} \operatorname{Cov}\left(\boldsymbol{x}_{i_{1}}^{\left(n_{1}\right)}, \boldsymbol{x}_{i_{2}}^{\left(n_{2}\right)}\right),
\end{aligned}
$$

where, in this manuscript, a covariance between two vectors/matrices $\boldsymbol{X}_{1}$ and $\boldsymbol{X}_{2}$ is defined as:

$$
\operatorname{Cov}\left(\boldsymbol{X}_{1}, \boldsymbol{X}_{2}\right)=\mathrm{E}\left[\left(\boldsymbol{X}_{1}-\mathrm{E}\left[\boldsymbol{X}_{1}\right]\right)\left(\boldsymbol{X}_{2}-\mathrm{E}\left[\boldsymbol{X}_{2}\right]\right)^{T}\right] .
$$

Assuming there are $N$ trials in total, all possible combination of the trials are summed as:

$$
\begin{aligned}
\sum_{\substack{n_{1}, n_{2}=1 \\
n_{1} \neq n_{2}}}^{N} C_{n_{1}, n_{2}} & =\sum_{\substack{n_{1}, n_{2}=1 \\
n_{1} \neq n_{2}}}^{N} \sum_{i_{1}, i_{2}=1}^{I} w_{i_{1}} w_{i_{2}} \operatorname{Cov}\left(\boldsymbol{x}_{i_{1}}^{\left(n_{1}\right)}, \boldsymbol{x}_{i_{2}}^{\left(n_{2}\right)}\right) \\
& =\boldsymbol{w}^{T} \boldsymbol{S} \boldsymbol{w} .
\end{aligned}
$$
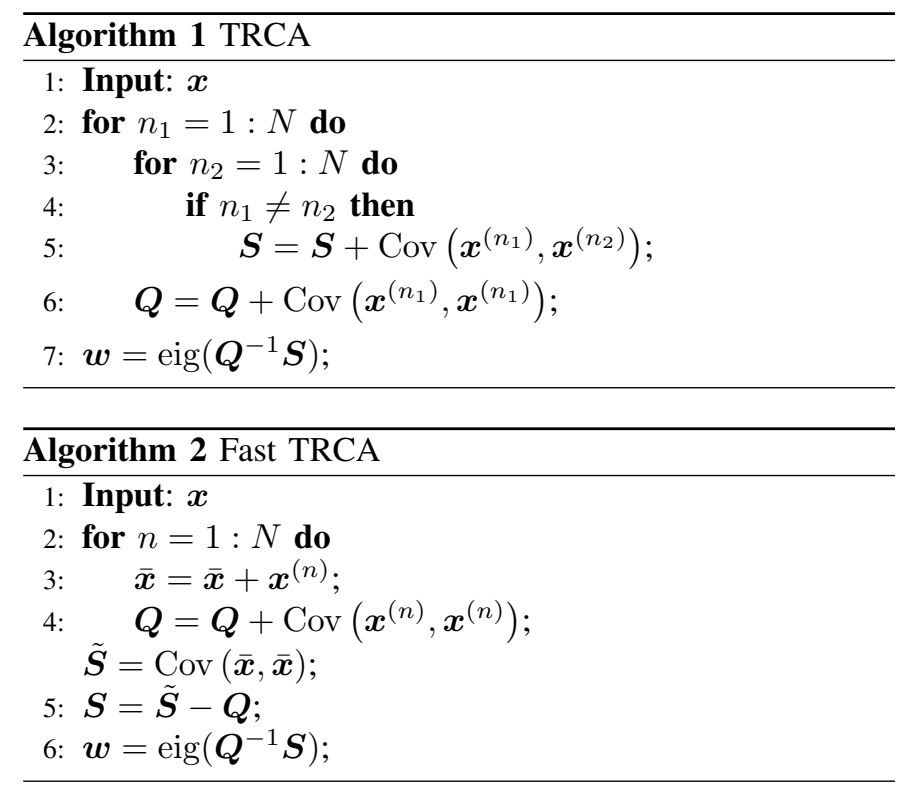

Here, the matrix $\boldsymbol{S}=\left(S_{i_{1} i_{2}}\right)_{1 \leq i_{1}, i_{2} \leq I}$ is defined as:

$$
S_{i_{1} i_{2}}=\sum_{\substack{n_{1}, n_{2}=1 \\ n_{1} \neq n_{2}}}^{N} \operatorname{Cov}\left(\boldsymbol{x}_{i_{1}}^{\left(n_{1}\right)}, \boldsymbol{x}_{i_{2}}^{\left(n_{2}\right)}\right)
$$

The optimal coefficient $\hat{w}$ to find task-related components can be obtained by maximizing the equation 3 . To obtain a finite solution, the sum of the variance of $\boldsymbol{y}^{(n)}$ is constrained as:

$$
\begin{aligned}
\sum_{n=1}^{N} \operatorname{Var}\left(\boldsymbol{y}^{(n)}\right) & =\sum_{n=1}^{N} \operatorname{Var}\left(\boldsymbol{w}^{T} \boldsymbol{x}^{(n)}\right) \\
& =\sum_{n=1}^{N} \sum_{i_{1}, i_{2}=1}^{I} w_{i_{1}} w_{i_{2}} \operatorname{Cov}\left(\boldsymbol{x}_{i_{1}}^{(n)}, \boldsymbol{x}_{i_{2}}^{(n)}\right) \\
& =\boldsymbol{w}^{T} \boldsymbol{Q} \boldsymbol{w} \\
& =1 .
\end{aligned}
$$

Here, the matrix $\boldsymbol{Q}=\left(Q_{i_{1}, i_{2}}\right)_{1 \leq i_{1}, i_{2} \leq I}$ is defined as:

$$
Q_{i_{1}, i_{2}}=\sum_{n=1}^{N} \operatorname{Cov}\left(\boldsymbol{x}_{i_{1}}^{(n)}, \boldsymbol{x}_{i_{2}}^{(n)}\right) .
$$

The constrained optimization problem can be solved as:

$$
\hat{\boldsymbol{w}}=\underset{\boldsymbol{w}}{\operatorname{argmax}} \frac{\boldsymbol{w}^{T} \boldsymbol{S} \boldsymbol{w}}{\boldsymbol{w}^{T} \boldsymbol{Q} \boldsymbol{w}} .
$$

The optimal coefficient vector is obtained as the eigenvector of the matrix $Q^{-1} \boldsymbol{S}$. The eigenvector corresponding to the largest eigenvalue $\hat{\boldsymbol{w}}$ is selected as the linear filter to extract task-related components.

\section{FASt TASK-RELATEd COMPONENT ANALYSIS}

The original method for solving TRCA requires multiple cross-covariance calculations whose computational cost increases exponentially as the number of trials increases. In Fig 1 a), trial pairs in solving TRCA are illustrated in a matrix 


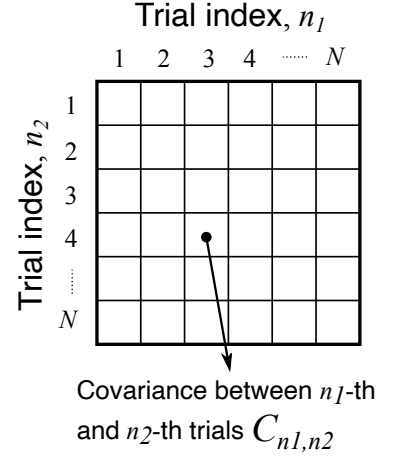

(a)
Original approach

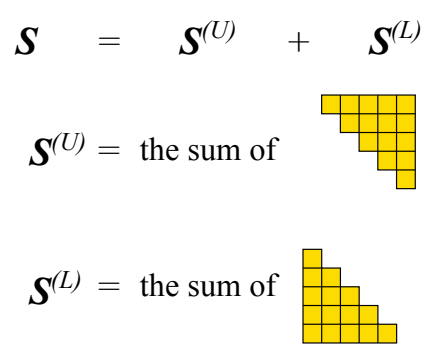

Proposed approach

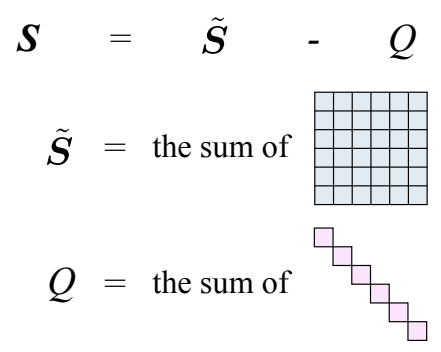

(b)

Fig. 1. The illustration of computational approaches in the original and proposed TRCA. (a) A matrix representation of pairs of trials. Each element $C_{n_{1}, n_{2}}$ indicates the covariance between the $n_{1}$-th and the $n_{2}$-th trials. (b) The matrix $S$ in the original TRCA is computed as the summation of the upper and lower covariance (i.e., $\boldsymbol{S}^{(U)}$ and $\boldsymbol{S}^{(L)}$ ) in the matrix. In the proposed TRCA, it can be computed by subtracting the diagonal covariance elements $\boldsymbol{Q}$ from the summation of all the covariance elements $\tilde{\boldsymbol{S}}$ in the matrix.

representation. Each element in the matrix represents the crosscovariance between the $n_{1}$-th and the $n_{2}$-th trials (i.e., $C_{n_{1}, n_{2}}$ in the equation 1 ).

In the original approach, all the elements except the diagonal ones need to be calculated and summed up to obtain $\boldsymbol{S}$. Although the equation 4 is written in a simplified form, its more precise formulation is:

$$
\begin{aligned}
S_{i_{1}, i_{2}}= & \sum_{n_{1}=1}^{N-1} \sum_{n_{2}=n_{1}+1}^{N} \operatorname{Cov}\left(\boldsymbol{x}_{i_{1}}^{\left(n_{1}\right)}, \boldsymbol{x}_{i_{2}}^{\left(n_{2}\right)}\right) \\
& +\sum_{n_{2}=1}^{N-1} \sum_{n_{1}=n_{2}+1}^{N} \operatorname{Cov}\left(\boldsymbol{x}_{i_{1}}^{\left(n_{1}\right)}, \boldsymbol{x}_{i_{2}}^{\left(n_{2}\right)}\right) .
\end{aligned}
$$

The first and second terms in this equation correspond to the sum of the elements in the upper and lower triangular parts of $S$, respectively, as shown in Fig 1.b). Therefore, the equation 8 can be rewritten as:

$$
\boldsymbol{S}=\boldsymbol{S}^{(U)}+\boldsymbol{S}^{(L)}
$$

where the elements of $\boldsymbol{S}^{(U)}$ and $\boldsymbol{S}^{(L)}$ are defined as:

$$
\begin{aligned}
S_{i_{1}, i_{2}}^{(U)} & =\sum_{n_{1}=1}^{N-1} \sum_{n_{2}=n_{1}+1}^{N} \operatorname{Cov}\left(\boldsymbol{x}_{i_{1}}^{\left(n_{1}\right)}, \boldsymbol{x}_{i_{2}}^{\left(n_{2}\right)}\right), \\
S_{i_{1}, i_{2}}^{(L)} & =\sum_{n_{2}=1}^{N-1} \sum_{n_{1}=n_{2}+1}^{N} \operatorname{Cov}\left(\boldsymbol{x}_{i_{1}}^{\left(n_{1}\right)}, \boldsymbol{x}_{i_{2}}^{\left(n_{2}\right)}\right),
\end{aligned}
$$

respectively. Since there are $2\left(\begin{array}{c}N \\ 2\end{array}\right)=N^{2}-N$ pairs of trials, the computational cost of $S$ with the equation 9 is $\mathcal{O}\left(N^{2}\right)$.

The $S$ can also be calculated by subtracting the sum of diagonal elements from the sum of all the covariance elements in the matrix as shown in Fig 1. b) as:

$$
\boldsymbol{S}=\tilde{\boldsymbol{S}}-\boldsymbol{Q}
$$

where the matrix $\tilde{\boldsymbol{S}}$ is defined as:

$$
\begin{aligned}
\tilde{S}_{i_{1}, i_{2}} & =\sum_{n_{1}=1}^{N} \sum_{n_{2}=1}^{N} \operatorname{Cov}\left(\boldsymbol{x}_{i_{1}}^{\left(n_{1}\right)}, \boldsymbol{x}_{i_{2}}^{\left(n_{2}\right)}\right) \\
& =\sum_{n_{1}=1}^{N} \sum_{n_{2}=1}^{N} \frac{1}{J}\left(\boldsymbol{x}_{i_{1}}^{\left(n_{1}\right)}-\mu_{i_{1}}^{\left(n_{1}\right)}\right)^{T}\left(\boldsymbol{x}_{i_{2}}^{\left(n_{2}\right)}-\mu_{i_{2}}^{\left(n_{2}\right)}\right) \\
& =\frac{1}{J} \sum_{n_{1}=1}^{N}\left(\boldsymbol{x}_{i_{1}}^{\left(n_{1}\right)}-\mu_{i_{1}}^{\left(n_{1}\right)}\right)^{T} \sum_{n_{2}=1}^{N}\left(\boldsymbol{x}_{i_{2}}^{\left(n_{2}\right)}-\mu_{i_{2}}^{\left(n_{2}\right)}\right) \\
& =\operatorname{Cov}\left(\overline{\boldsymbol{x}}_{i_{1}}, \overline{\boldsymbol{x}}_{i_{2}}\right) .
\end{aligned}
$$

Here, $\mu_{i}^{(n)}$ is an average of $x_{i}^{(n)}$ across sample points in a data epoch and $\overline{\boldsymbol{x}}$ is the summation of $\boldsymbol{x}^{(n)}$ across trials as:

$$
\begin{array}{r}
\mu_{i}^{(n)}=\frac{1}{J} \sum_{j=1}^{J} x_{i j}^{(n)}, \\
\overline{\boldsymbol{x}}_{i}=\sum_{n=1}^{N} \boldsymbol{x}_{i}^{(n)} .
\end{array}
$$

The equation 13 indicates that $\tilde{\boldsymbol{S}}$ can be simply computed as the auto-covariance of $\overline{\boldsymbol{x}}$, whose computational cost does not depend on the number of trials. The computational cost of $S$ can be reduced to $\mathcal{O}(1)$ from $\mathcal{O}\left(N^{2}\right)$ by introducing the new computational approach. Since the computational cost of $\boldsymbol{Q}$ is still proportional to the number of trials (i.e., $N$ ), the total computational time to solve TRCA would also be proportional to the number of trials (See Algorithm 2).

\section{COMPUTER Simulation}

To validate the effectiveness of the proposed reformulation in reducing computational time, we conducted numerical simulations with synthetic data. The synthetic data were designed to simulate the application of TRCA to biological signals such as fNIRS and ERP data. We prepared three-way tensors $\boldsymbol{X} \in \mathbb{R}^{I \times J \times N}$ with different numbers of data dimensions $(I=[4,16,64])$, data lengths $(J=[1000,2000,4000,8000]$; $2,4,8$, and $16 \mathrm{~s}$ at a sampling rate of $500 \mathrm{~Hz}$ ), and the number of data segments $(N=[2,4,8, \ldots, 512,1024])$ to estimate the computational time with different data formats. A sinusoidal 

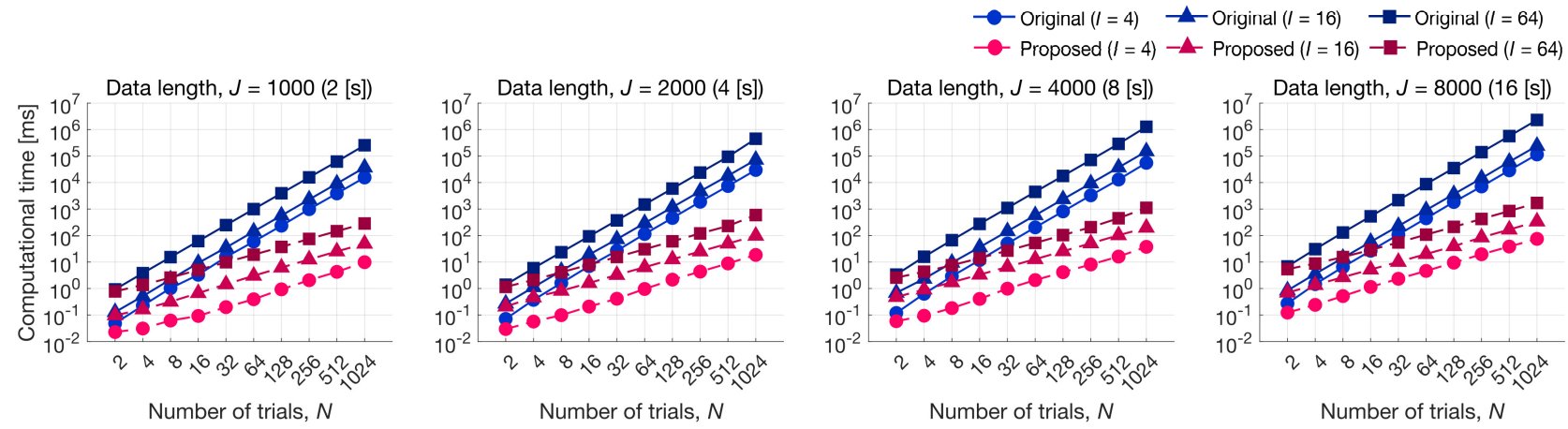

Fig. 2. The computational time in the original and proposed TRCA as functions of different numbers of trials $(N)$ at various data dimensions $(I)$ and data lengths $(J)$ using synthetic data. The horizontal and vertical axes are presented in logarithmic scales with bases of 2 and 10 , respectively.

signal $s \in \mathbb{R}^{J}$ at arbitrary frequency selected randomly from 0 to $60 \mathrm{~Hz}$ was first generated as a task-related component, and then linearly projected with a random coefficient $a \in \mathbb{R}^{I}$ to generate multi-dimensional data $\boldsymbol{x} \in \mathbb{R}^{I \times J}$ as $\boldsymbol{x}=\boldsymbol{a \boldsymbol { s } ^ { T }}$. To synthesize multi-trial data, different random noises $\boldsymbol{e}_{n} \in \mathbb{R}^{I \times J}$ were added to $\boldsymbol{x}$ in each trial as $\boldsymbol{X}_{n}=\boldsymbol{x}+\boldsymbol{e}_{n}$. The computational time was estimated by inputting the multi-dimensional data $\boldsymbol{X}$ into the original and the proposed TRCA. Note that the computational time is independent from the signals itself as long as the dimensionality is consistent. The computational time was measured by using tic/toc functions in MATLAB. Because a single computation to solve the original/proposed TRCA is too fast to obtain accurate computational time by tic/toc functions, we measured the elapsed time over $K$ iterations and divided the obtained elapsed time by $K$ to estimate the computational time for a single computation under each parameter setting (i.e., $I, J$, and $N$ ). For the proposed TRCA, $K$ was set to 10,000 regardless of parameters. For the original TRCA, $K$ was $10,000,100$, and 10 when $N=[2$, $4,8],[16,32,64]$, and $[128,256,512,1024]$, respectively, to avoid excessive computational time. The simulation was conducted using MATLAB R2021a on Windows 11 with an Intel Core i7-7700HQ $2.80 \mathrm{GHz}$ processor.

Fig. 2 shows the simulation results. In all the parameter settings, the computational time and the number of trials were presented in logarithmic scales with bases of 10 and 2 , respectively. In general, as the number of data dimensions (i.e., $I$ ), data length (i.e., $J$ ) and the number of trials (i.e., $N$ ) increased, so did the computational time. Importantly, while the computational time for the proposed approach increased with the number of trials, it was slower than the one for the original approach. We calculated the slopes of the curves in the logarithmic scale. Because the computational complexity of the original and proposed approaches should be proportional to $N^{2}$ and $N$, respectively, theoretically the slopes for the original approach should be approximately double those of the proposed approach on the logarithmic scale. The averaged slopes across different numbers of data dimensions and data lengths for the original and the proposed approaches were 0.6127 and 0.2973 , respectively, proving this prediction.

We also investigated whether the two approaches could extract the identical results (i.e., task-related components).
TABLE I

THE AVERAGE MEAN SQUARE ERROR OF TWO COMPONENTS EXTRACTED BY THE ORIGINAL AND THE PROPOSED TRCA.

\begin{tabular}{lcccc}
\hline \hline \# of dims & \multicolumn{4}{c}{ Data lengths, $J$} \\
\cline { 2 - 5 }$I$ & 1000 & 2000 & 4000 & 8000 \\
\hline 4 & $2.24 \mathrm{e}-17$ & $1.91 \mathrm{e}-17$ & $9.17 \mathrm{e}-18$ & $7.82 \mathrm{e}-18$ \\
16 & $2.12 \mathrm{e}-17$ & $2.25 \mathrm{e}-17$ & $1.12 \mathrm{e}-17$ & $8.71 \mathrm{e}-18$ \\
64 & $1.21 \mathrm{e}-16$ & $6.18 \mathrm{e}-17$ & $5.36 \mathrm{e}-17$ & $2.89 \mathrm{e}-17$ \\
\hline \hline
\end{tabular}

Table [ shows the mean square errors between the task-related components extracted by the original and proposed TRCA. The errors between the two approaches were smaller than $10^{-15}$ under all the parameter settings. In other words, two approaches produced nearly identical components. The nonzero mean squared error might be due to a floating-point exception. This result confirmed that the proposed reformulation significantly reduced the computational time to solve TRCA without distorting its results.

The proposed approach has a great potentials to be used in neuro-imaging and neural engineering studies. By introducing the proposed reformulation, the extended versions of TRCA that require high computational cost such as xTRCA and gTRCA would become significantly computationally inexpensive [5]-[7]. Furthermore, as mentioned in the Introduction, TRCA has been widely used in the implementation of BCIs [3], [10]. In recent BCI studies, transfer learning approaches have been used to train subject-independent machine-learning models with zero-/reduced-calibration processes [11]-[16]. TRCA has also been used to extract domain-independent features (e.g., SSVEPs and P300s) to transfer them across different domains such as subjects, sessions, and EEG devices. [17]-[19]. Because such transfer-learning approaches typically deal with large datasets, the proposed reformulation could significantly reduce the number of matrix multiplications (i.e., computational time) required to solve TRCA.

\section{CONClusion}

This letter introduced a reformulation for TRCA to reduce the number of matrix multiplications used in the calculation. The computer simulation confirmed that the proposed reformulation could reduce the computational complexity from $\mathcal{O}\left(N^{2}\right)$ to $\mathcal{O}(N)$, leading to a wide range of practical applications in neuroimaging and neural engineering studies. 


\section{REFERENCES}

[1] H. Tanaka, T. Katura, and H. Sato, "Task-Related Component Analysis for Functional Neuroimaging and Application to Near-Infrared Spectroscopy Data," NeuroImage, vol. 64, pp. 308-327, 2013.

[2] H. Tanaka, T. Katura, and H. Sato, "Task-related oxygenation and cerebral blood volume changes estimated from NIRS signals in motor and cognitive tasks," Neuroimage, vol. 94, pp. 107-119, Jul. 2014.

[3] M. Nakanishi, Y. Wang, X. Chen, Y. T. Wang, X. Gao, and T. P. Jung, "Enhancing Detection of SSVEPs for a High-Speed Brain Speller Using Task-Related Component Analysis," IEEE Trans. Biomed. Eng., vol. 65, no. 1, pp. 104-112, 2018.

[4] C. M. Wong, B. Wang, Z. Wang, K. F. Lao, A. Rosa, and F. Wan, "Spatial filtering in SSVEP-Based BCIs: Unified framework and new improvements," IEEE Trans. Biomed. Eng., vol. 67, no. 11, pp. 30573072, Nov. 2020.

[5] H. Tanaka and M. Miyakoshi, "Cross-correlation task-related component analysis (xTRCA) for enhancing evoked and induced responses of eventrelated potentials," Neuroimage, vol. 197, pp. 177-190, 2019.

[6] H. Tanaka, "Group task-related component analysis (gTRCA): a multivariate method for inter-trial reproducibility and inter-subject similarity maximization for EEG data analysis," Sci. Rep., vol. 10, no. 1, p. 84, Jan. 2020.

[7] H. Tanaka, T. Katura, and H. Sato, Data Analysis Method for Neuroimaging Data: Task-Related Component Analysis and Its Applications to fNIRS Data. Singapore: Springer Singapore, 2020, pp. 149-173.

[8] T. P. Jung, S. Makeig, M. Westerfield, J. Townsend, E. Courchesne, and T. J. Sejnowski, "Analysis and visualization of single-trial event-related potentials," Hum. Brain Mapp., vol. 14, no. 3, pp. 166-185, Nov. 2001.

[9] K.-J. Chiang, M. Nakanishi, and T.-P. Jung, "Statistically optimized spatial filtering in decoding Steady-State visual evoked potentials based on Task-Related component analysis," Conf. Proc. IEEE Eng. Med. Biol. Soc., vol. 2020, pp. 3070-3073, Jul. 2020.

[10] M. Nakanishi, Y. Wang, and T.-P. Jung, "Session-to-session transfer in detecting steady-state visual evoked potentials with individual training data," in International Conference on Augmented Cognition. Springer, 2016, pp. 253-260.
[11] P. Yuan, X. Chen, Y. Wang, X. Gao, and S. Gao, "Enhancing performances of SSVEP-based brain-computer interfaces via exploiting intersubject information," J. Neural Eng., vol. 12, no. 4, p. 046006, 2015.

[12] N. R. Waytowich, J. Faller, J. O. Garcia, J. M. Vettel, and P. Sajda, "Unsupervised adaptive transfer learning for steady-state visual evoked potential brain-computer interfaces," in 2016 IEEE International Conference on Systems, Man, and Cybernetics (SMC). IEEE, 2016, pp. $004135-004140$.

[13] B. Liu, X. Chen, X. Li, Y. Wang, X. Gao, and S. Gao, "Align and pool for EEG headset domain adaptation (ALPHA) to facilitate dry electrode based SSVEP-BCI," IEEE Trans. Biomed. Eng., 2021.

[14] C. M. Wong, Z. Wang, A. C. Rosa, C. L. P. Chen, T.-P. Jung, Y. Hu, and F. Wan, "Transferring Subject-Specific knowledge across stimulus frequencies in SSVEP-Based BCIs," IEEE Trans. Autom. Sci. Eng., vol. 18, no. 2, pp. 552-563, Apr. 2021.

[15] C. M. Wong, Z. Wang, B. Wang, K. F. Lao, A. Rosa, P. Xu, T.-P. Jung, C. P. Chen, and F. Wan, "Inter-and intra-subject transfer reduces calibration effort for high-speed SSVEP-based BCIs," IEEE Trans. Neural Syst. Rehabil. Eng., vol. 28, no. 10, pp. 2123-2135, 2020.

[16] P.-J. Kindermans, M. Tangermann, K.-R. Müller, and B. Schrauwen, "Integrating dynamic stopping, transfer learning and language models in an adaptive zero-training ERP speller," J. Neural Eng., vol. 11, no. 3, p. 035005, Jun. 2014.

[17] M. Nakanishi, Y.-T. Wang, C.-S. Wei, K.-J. Chiang, and T.-P. Jung, "Facilitating calibration in High-Speed BCI spellers via leveraging CrossDevice shared latent responses," IEEE Trans. Biomed. Eng., vol. 67, no. 4, pp. 1105-1113, Apr. 2020.

[18] K.-J. Chiang, C.-S. Wei, M. Nakanishi, and T.-P. Jung, "Boosting template-based SSVEP decoding by cross-domain transfer learning," $J$. Neural Eng., vol. 18, no. 1, p. 016002, 2021.

[19] T. Wang, P. Liu, X. An, Y. Ke, J. Xu, M. Xu, L. Kong, W. Liu, and D. Ming, "Modeling strategies and spatial filters for improving the performance of p300-speller within and across individuals," in 2019 IEEE International Conference on Computational Intelligence and Virtual Environments for Measurement Systems and Applications (CIVEMSA), Jun. 2019, pp. 1-5. 